\title{
A SXES and CL Spectral Library for the Analysis of Rare Earth Elements
}

\author{
C.M. MacRae ${ }^{1}$, N.C. Wilson ${ }^{1}$, A. Torpy ${ }^{1}$, H. Takahashi ${ }^{2}$, T. Murano ${ }^{2}$ and M. Takaura ${ }^{2}$ and C. Lenz ${ }^{1,3}$ \\ ${ }^{1 .}$ CSIRO Mineral Resources, Private Bag 10, Clayton South, VIC, Australia \\ 2. JEOL Ltd., 3-1-2 Musashino, Akishima, Tokyo, Japan \\ 3. Institut für Mineralogie und Kristallographie, Universität Wien, Austria
}

Cathodoluminescence (CL) and soft $\mathrm{x}$-ray emission (SXE) spectrometers are both sensitive to chemical bonding and offer insight into the local environment of rare earth ions. Rare earth (RE) containing minerals and materials often emit strong CL associated with the RE ion. The spectra are often complex and the relative peak intensities, position and width can all vary with both host composition and structure. Similarly SXE spectra of RE containing compounds show peak movement from changes in the host lattice and the structure. Since both can now be measured then they should both be used to offer extra insight into the RE environment. To investigate this we have collected a series of materials containing RE elements in different structures. These include the REE containing phosphates, fluorides manufactured by Charles M. Taylor, glasses made by University of Edinburgh, and various REE containing oxides and borides. The collected spectra will be added to the luminescence database [1] and made available through the website [2] and be included in the distribution set of spectra given out with OpticalFit [2].

RE containing compounds are often beam sensitive, so stage mapping was employed to collect the CL spectra with no damage and the SXES spectra were collected by either mapping or moving the sample under the beam during acquisition. The reference CL spectra were collected at 20kV, 10nA, dwell time per pixel $25 \mathrm{~ms}$ and the reference SXE spectra were collected at $5 \mathrm{kV}$ with $10 \mathrm{~s}$ dwell.

In Figure 1, europium CL spectra are shown from three different structures. The Eu lines at $1.95-$ $2.16 \mathrm{eV}$ have been attributed to ${ }^{5} \mathrm{D}_{0}-{ }^{7} \mathrm{~F}_{2}$ transitions, with these peaks in the glass matrix are broadened. In addition, across the wider spectrum the relative peak intensities vary depending upon the host matrix.

Figure 2, shows a series of SXE spectra from orthphosphates containing REE's. The P L series of peaks can be seen around $120 \mathrm{eV}$ and a second order carbon reflection at $138 \mathrm{eV}$. A closer examination, Figure 3, reveals several eV of peak movement in the P L line depending upon the associated RE cation.

\section{References:}

[1] C.M. MacRae and N.C. Wilson, Microscopy and Microanalysis, 14, 184-204, 2008

[2] CSIRO luminescence database www.csiro.au/luminescence 


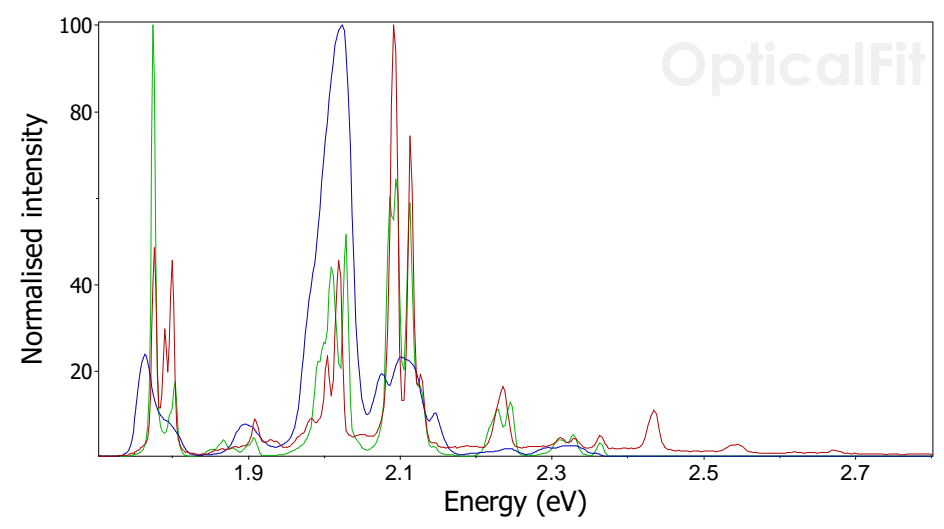

Figure 1. Comparison of cathodoluminescence spectra from Eu fluoride (red), Eu Aluminium silicate (blue) and $\mathrm{Eu}$ orthophosphate (green) showing significant peak broadening in the glass and sharper peaks observed in the orthophosphate.

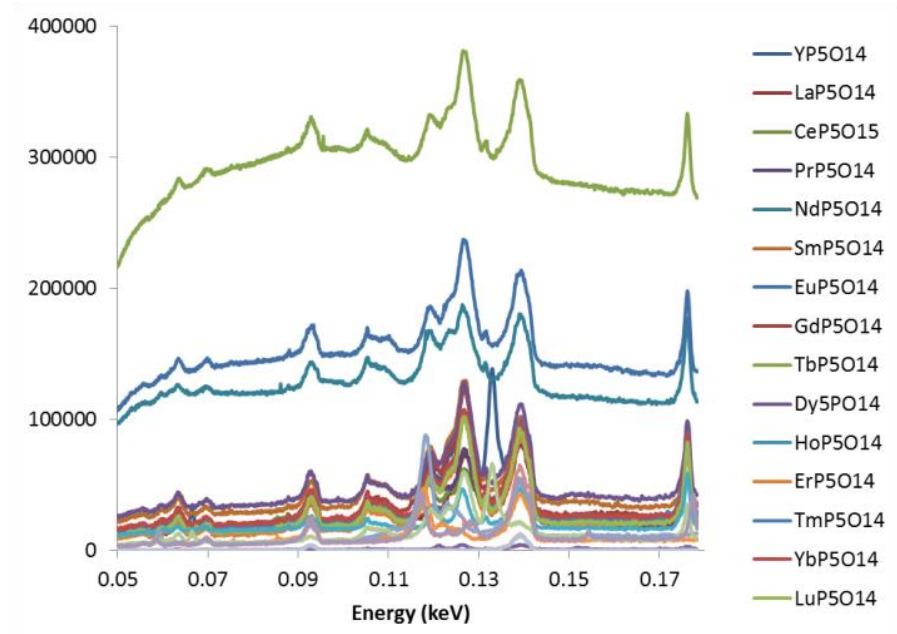

Figure 2. Comparison of SXE spectra from a series of orthphosphates containing REE's. The strong CL emitters show an elevated background. The P L series of peaks can be seen around $120 \mathrm{eV}$ and a second order carbon reflection at $138 \mathrm{eV}$.

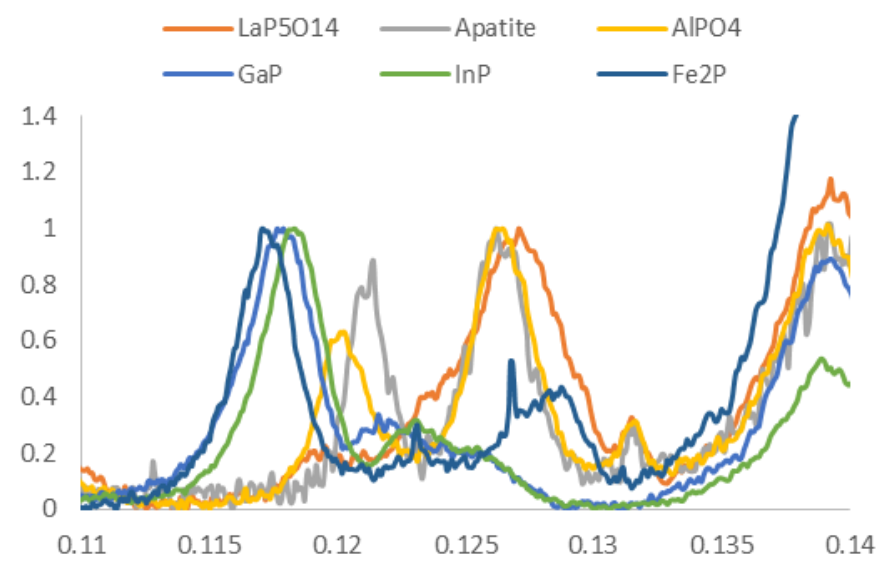

Figure 3. Zoomed in view of spectra selected from figure 2, showing the movement in the P L series lines. 